\title{
The Effectiveness of Hamstring Stretching with Proprioceptive Neuromuscular Facilitation versus Jack-Knife Stretching for Individuals with Hamstring Tightness
}

\author{
Jae-Seop Oh, PT, Ph.D ${ }^{1}$; Min-Hyeok Kang, PT, Ph.D ${ }^{2}$ \\ ${ }^{1}$ Department of Physical Therapy, College of Biomedical Science and Engineering, Inje University, Gimhae, South Korea \\ ${ }^{2}$ Department of Physical Therapy, College of Health Sciences, Catholic University of Pusan, Busan, South Korea
}

Background Jack-knife stretching is a newly developed static hamstring stretching exercise. However, no studies have compared this stretching exercise to hamstring stretching with proprioceptive neuromuscular facilitation (PNF).

Purpose This study compared the effects of PNF hamstring stretching and jack-knife stretching on hamstring flexibility.

Study design Randomized controlled clinical trial

Methods Twenty-four adults with bilateral hamstring tightness were randomly assigned to the PNF hamstring stretching or jack-knife stretching group. All participants performed PNF hamstring stretching or jack-knife stretching for $150 \mathrm{~s}$. Before and after hamstring stretching, the participants completed the active knee extension (AKE), active straight leg raise (ASLR), and finger-tofloor distance (FFD) tests. Lumbar flexion and the anterior pelvic tilt angle were measured during the FFD test.

Results Both hamstring stretching exercises increased the knee extension angle during the AKE test, hip flexion angle during the ASLR test, and lumbar flexion and anterior pelvic tilt angle during the FFD test while decreasing the FFD value $(p<0.05)$. However, no significant betweengroup differences were found $(p>0.05)$.

Conclusions PNF hamstring stretching and jack-knife stretching have similar beneficial effects on hamstring flexibility.

Key words Hamstring stretching; Hamstring tightness; Pelvic movement; Proprioceptive neuromuscular facilitation; Static stretching.
J Musculoskelet Sci Technol 2021; 5(1): 14-20 Published Online Jun 30, 2021 pISSN 2635-8573 eISSN 2635-8581
Article History Received 8 Mar 2021 Revised 25 Mar 2021 (1st) Revised 29 Mar 2021 (2nd) Accepted 29 Mar 2021

\section{CONTACT}
kmhyuk01@gmail.com Min-Hyeok Kang, Department of Physical Therapy, College of Health Sciences, Catholic University of Pusan, Busan, South Korea This is an Open-Access article
distributed under the terms of
the Creative Commons Att-
ribution Non-Commercial Li-
cense (http://creativecommons.
org/licenses/by-nc/4.0) which
permits unrestricted non-co-
mmercial use, distribution,
and reproduction in any me-
dium, provided the original
work is properly cited.

\section{INTRODUCTION}

The hamstring muscle group comprises the semimembranous, semitendinous, and biceps femoris muscles, which commonly originate from the ischial tuberosity. ${ }^{1}$ Because of this common muscle origin, contraction of the hamstring muscles results in pelvic posterior tilt as well as hip extension and knee flexion. ${ }^{2,3}$ In addition, because the pelvis is connected to the lumbar spine, pelvic anterior and posterior tilt causes decreased and increased lumbar flexion, respectively. ${ }^{2,3}$ Therefore, tight hamstring muscles leading to pelvic posterior tilt could cause excessive lumbar flexion, which is a risk factor for low back pain. ${ }^{1-4}$

Hamstring stretches with proprioceptive neuromuscular facilitation (PNF) are commonly used to address hamstring muscle tightness in clinical settings. Hamstring stretching with PNF using the hold-relax (HR) technique is a method in which relaxation is induced after performing isometric contraction to the point where resistance is felt in the hamstring muscles. ${ }^{5-7}$ Activation of the Golgi tendon organ 
(GTO) after isometric contraction of the hamstring muscles leads to autogenic inhibition, which can improve hamstring flexibility. ${ }^{8-11}$ A previous study found that PNF hamstring stretches decreased lumbar flexion while increasing hip flexion during lifting tasks requiring lumbar flexion movements. Therefore, PNF hamstring stretches may influence movements in both the lower extremities and lumbar spine. ${ }^{8}$

During static hamstring stretching, tightness is released in the hamstring muscles while hip flexion and/or knee extension positions are held in the direction that lengthens the hamstring muscles. ${ }^{12,13}$ Jack-knife stretching is a newly suggested static hamstring stretching method. ${ }^{14}$ In this stretching method, a squat position is assumed and the ankles are held with both hands. A previous study demonstrated that both short-term and long-term jack-knife stretching improved hamstring flexibility, as assessed via the finger-to-floor distance (FFD) test, sit and reach test (SRT), active knee extension (AKE) test, passive straight leg raising (PSLR) test, and active straight leg raising (ASLR) test. ${ }^{14,15}$

Although both PNF hamstring stretching and static hamstring stretching can effectively improve hamstring flexibility, it is unclear whether one of these exercises is superior. ${ }^{5,16-18}$ While some previous studies showed no significant differences in hamstring flexibility between the two hamstring stretching exercises, ${ }^{16,17}$ others demonstrated that PNF hamstring stretching was superior to static hamstring stretching in terms of improved hamstring flexibility. ${ }^{5,18}$ Although these previous studies compared the effects of static hamstring stretching to PNF hamstring stretching, ${ }^{5,16-18}$ they did not consider the newly-developed jack-knife stretching method. Comparing the PNF hamstring and jack-knife stretching methods could help clinicians determine the best strategy for improving hamstring flexibility and decreasing mechanical stress due to excessive lumbar flexion caused by hamstring tightness. Thus, in the present study, we compared the PNF hamstring and jack-knife stretching methods in terms of hamstring flexibility and lumbo-pelvic movements.

\section{METHODS}

\section{Subjects}

Twenty-four adults with bilateral hamstring tightness participated in this study. The bilateral knee extension range of motions (ROMs) were less than $160^{\circ}$ for all participants in the AKE test. We excluded subjects who had undergone surgery of the knee or lumbar spine, been diagnosed with a deformity of the spine or lower extremities, or who reported pain in the last 6 months. ${ }^{8,9}$ Sample size was determined based on large effect size $(d=0.8), 80 \%$ statistical power, and 0.05 of significance level to detect change in hamstring flexibility between pre- and post-exercises for each group.

Subjects were randomly assigned to the PNF hamstring stretching group (7 men and 5 women) or the jack-knife stretching group ( 7 men and 5 women). All subjects provided written informed consent and the study protocols were approved by the Institutional Research Review Committee of Inje University.

\section{Hamstring length test: AKE test}

For the AKE test, the test leg of each subject was placed in the $90^{\circ}$ hip flexion position with the other leg supported on the table in the supine position. The subjects were asked to extend their knee on the test side with relaxed ankle position until they felt a maximum stretch. ${ }^{8,15}$ An examiner monitored compensated lumbar extension movement with visual inspection and recorded the knee extension angle using an inclinometer application (Plaincode Software Solutions, Stephanskirchen, Germany) at the end-range point of knee extension. ${ }^{15}$ We calculated the knee extension angle at the tibia position with respect to the line parallel to the examination table. AKE tests were performed on both sides, and repeated three times with $30 \mathrm{~s}$ of rest period between repetitions for each leg. The mean value from the three trials was used for data analysis.

\section{Hamstring length test: ASLR test}

We used the ASLR test to examine changes in the hip flexion ROM after stretching. While in the supine position, the subjects were asked to flex their hip and extend their knee with relaxed ankle position on the test side until they felt a maximum stretch. ${ }^{5,15}$ An examiner monitored compensated lumbar extension movement with visual inspection and placed a smartphone on the subject's anterior thigh and recorded the end-range of the hip flexion angle using the inclinometer application. ${ }^{15}$ ASLR tests were performed on both sides, and repeated three times with $30 \mathrm{~s}$ of rest period between repetitions for each leg. The mean value from the three trials was used for data analysis.

\section{Hamstring length test: FFD test}

We used the FFD test to measure not only hamstring flexibility but also lumbo-pelvic movements. To monitor lumbo-pelvic movements during the FFD test, two gyroscope sensors (4D-MT Motion Sensor, Relive Co., Ltd., Gimhae, Republic of Korea) were attached at the first and second lumbar vertebrae at the mid-point between the posterior superior iliac spine and the pubic symphysis. ${ }^{15}$ 
While in the standing position, the subjects were asked to bend forward to the best of their ability. During the FFD test, the subjects were instructed to maintain an extended knee position. An examiner measured the distance between the floor and the subject's fingers using a tape measure. ${ }^{14,15}$ The lumbar flexion and anterior pelvic tilt were also recorded during the FFD test using gyroscope sensors. ${ }^{15}$ The FFD test was repeated three times with $30 \mathrm{~s}$ of rest period between repetitions and the mean value was used for data analysis.

Hamstring stretching protocol: PNF hamstring stretching

Subjects in the PNF hamstring stretching group performed only PNF hamstring stretching, while those in the jack-knife stretching group performed only jack-knife stretching. For PNF hamstring stretching, the subjects positioned their leg in the $90^{\circ}$ hip and knee flexion position and placed their ankle on the shoulder of an examiner. The examiner passively extended the knee until they felt the limit and held this position for $8 \mathrm{~s}$. Then the subjects were asked to perform isometric knee flexion with $50 \%$ of maximal force for $8 \mathrm{~s}$. The examiner again passively extended the subject's knee until a new limit was encountered (over a period of $6 \mathrm{~s}$ ) and held this position for $8 \mathrm{~s}$ (Figure 1A). This procedure was repeated five times. PNF hamstring stretching was performed on both sides, and the first side was selected randomly.

\section{Hamstring stretching protocol: Jack-knife stretching}

For jack-knife stretching, the subjects assumed a full squat position and grasped both ankles. They were asked to perform maximum knee extension while contacting their chest with their thighs and holding their ankles. The subjects maintained a maximum knee extension position for $10 \mathrm{~s}$ and then returned to the initial position (Figure 1B). This procedure was repeated 15 times with a 10 -s rest period between trials.

\section{Procedures}

All subjects performed the AKE, ASLR, and FFD tests in a randomized order, followed by hamstring stretching. After hamstring stretching according to group membership, all tests were repeated.

\section{Statistical analysis}

To compare the effects of the two hamstring stretching exercises on the dependent variables, we conducted a twoway repeated ANOVA with group and time as the main factors using PASW software ver. 18.0 (SPSS, Inc., Chicago, IL, USA). The general characteristics and baseline data from the two groups were compared using independent $t$ tests. The significance level was set at 0.05 . When main effects and/or interactions were found, we performed post hoc analyses using the Bonferroni correction.

\section{RESULTS}

We found no significant differences in age, height, body weight, or baseline data between the two groups $(p>0.05$; Table 1).

We found significant main effects of time for all dependent variables (FFD-distance: $p=0.001, \mathrm{~F}=21.590$; FFDanterior pelvic tilt: $p<0.001, \mathrm{~F}=27.899$; FFD-lumbar flexion: $p=0.004, \mathrm{~F}=12.912$; AKE test-right side: $p<0.001, \mathrm{~F}=67.739$; AKE test-left side: $p<0.001, \mathrm{~F}=44.294$; ASLR-right side: $p<0.001, \mathrm{~F}=118.973$; ASLR-left side: $p<0.001, \mathrm{~F}=152.390$; Table 2). However, there were no significant group-by-time
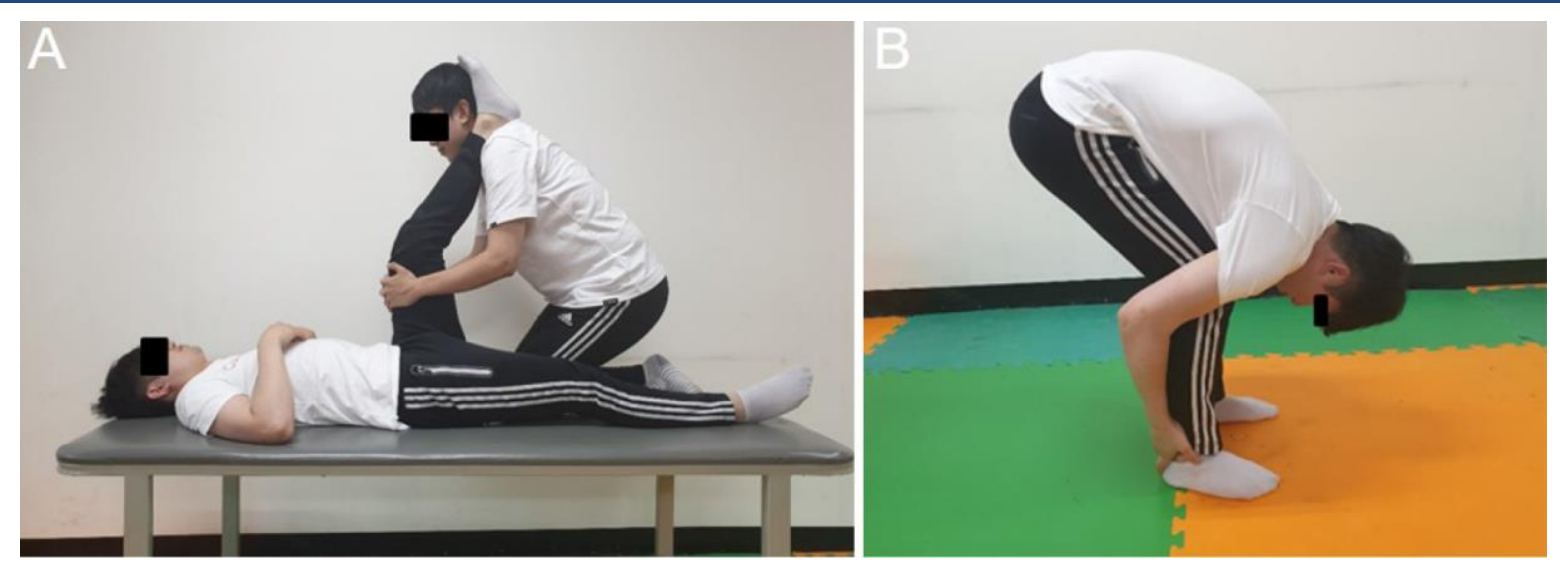

Figure 1. PNF hamstring stretching (A) and jack-knife stretching (B). Abbreviation: PNF, proprioceptive neuromuscular facilitation. 
Table 1. General characteristics and baseline data from the two hamstring stretching groups

\begin{tabular}{cccc}
\hline Characteristics & PNF hamstring stretching group & Jack-knife stretching group & $P$ value \\
\hline Gender (male/female) & $(N=12)$ & $(N=12)$ & None \\
Age (years) & $7 / 5$ & $7 / 5$ & 0.715 \\
\hline Body mass $(\mathrm{kg})$ & $21.33 \pm 1.97$ & $21.67 \pm 2.42$ & 0.203 \\
Height $(\mathrm{cm})$ & $65.25 \pm 12.93$ & $72.58 \pm 14.41$ & 0.607 \\
\hline FFD test - distance $(\mathrm{cm})$ & $167.17 \pm 9.33$ & $169.08 \pm 8.63$ & 0.958 \\
\hline FFD test - anterior pelvic tilt $\left(^{\circ}\right)$ & $6.03 \pm 11.09$ & $6.29 \pm 13.09$ & 0.361 \\
\hline FFD test - lumbar flexion $\left(^{\circ}\right)$ & $55.96 \pm 16.15$ & $62.76 \pm 19.39$ & 0.902 \\
AKE test - right side $\left(^{\circ}\right)$ & $96.93 \pm 13.07$ & $96.09 \pm 19.25$ & 0.513 \\
AKE test - left side $\left(^{\circ}\right)$ & $55.42 \pm 9.71$ & $52.67 \pm 10.55$ & 0.670 \\
ASLR - right side $\left({ }^{\circ}\right)$ & $53.03 \pm 9.47$ & $54.61 \pm 8.46$ & 0.551 \\
ASLR - left side $\left(^{\circ}\right)$ & $55.22 \pm 6.27$ & $57.92 \pm 14.10$ & 0.648 \\
\hline
\end{tabular}

Data are expressed as mean \pm standard deviation.

Abbreviation: AKE, active knee extension; ASLR, active straight leg raising; FFD, finger-to-floor distance; PNF, proprioceptive neuromuscular facilitation.

interactions (FFD-distance: $p=0.607, \mathrm{~F}=0.281$; FFD-anterior pelvic tilt: $p=0.240, \mathrm{~F}=1.542$; FFD-lumbar flexion: $p=0.288$ $\mathrm{F}=1.249$; AKE test-right side: $p=0.340, \mathrm{~F}=0.994$; AKE testleft side: $p=0.957, \mathrm{~F}=0.003$; ASLR-right side: $p=0.990$, $\mathrm{F}<0.001$; ASLR-left side: $p=0.856, \mathrm{~F}=0.035$; Table 2 ) or main effects of group (FFD-distance: $p=0.929, \mathrm{~F}=0.008$; FFD-anterior pelvic tilt: $p=0.504, \mathrm{~F}=0.477$; FFD-lumbar flexion: $p=0.883, \mathrm{~F}=0.023$; AKE test-right side: $p=0.366$, $\mathrm{F}=0.888$; AKE test-left side: $p=0.707, \mathrm{~F}=0.149$; ASLR-right side: $p=0.558, \mathrm{~F}=0.364$; ASLR-left side: $p=0.699, \mathrm{~F}=0.158$; Table 2) for any of the dependent variables.

In the post-hoc analysis, we found significant increases in anterior pelvic tilt (PNF hamstring stretching group: $p=$ 0.003, jack-knife stretching group: $p=0.008$ ) and lumbar flexion (PNF hamstring stretching group: $p=0.011$, jackknife stretching group: $p=0.014)$, together with significant decreases in distance (PNF hamstring stretching group: $p=0.001$, jack-knife stretching group: $p=0.012$ ) during the FFD test in both groups. In the AKE test, the AKE angle significantly increased after both stretching exercises on the right (PNF hamstring stretching group: $p=0.001$, jack-knife stretching group: $p<0.001$ ) and left (PNF hamstring stretching group: $p<0.001$, jack-knife stretching group: $p<0.001$ ) sides. In addition, the hip flexion angle during the ASLR test increased significantly after both stretching exercises on the right (PNF hamstring stretching group: $p<0.001$, jackknife stretching group: $p<0.001)$ and left (PNF hamstring stretching group: $p<0.001$, jack-knife stretching group: $p<$
$0.001)$ sides.

\section{DISCUSSION}

The present findings demonstrate that both the PNF hamstring stretching and jack-knife stretching techniques effectively improved hamstring flexibility and anterior pelvic tilt movements, and that the stretching exercises had similar effects on hamstring flexibility and pelvic movement.

Theoretically, PNF hamstring stretching improves hamstring flexibility via a type of neural inhibition called autogenic inhibition. ${ }^{8-11}$ During isometric contraction of a muscle, the GTO detects increased tension in the tendon and sends this information to the spinal cord, which leads to relaxation of the agonist muscle via autogenic inhibition. ${ }^{19,20}$ In the case of static hamstring stretching, holding the limb at the end-range of the ROM changes the viscoelastic properties of the muscle, which modulates the length-tension relationship and consequently improves muscle flexibility. ${ }^{18,21,22}$ Together, these physiological mechanisms may affect elongation of the hamstring muscles during both PNF hamstring stretching and jack-knife stretching, resulting in the decreased distance during the FFD test and increased knee extension and hip flexion angles during the AKE test and ASLR test, respectively, observed in the present study.

We found increased anterior pelvic tilt and lumbar flexion angles during the FFD test after both stretching exercises, 
Table 2. Descriptive data for the dependent variables in the two hamstring stretching groups

\begin{tabular}{|c|c|c|c|c|c|}
\hline \multirow{2}{*}{ Measures } & \multirow{2}{*}{ Pre-stretching } & \multirow{2}{*}{ Post-stretching } & \multicolumn{3}{|c|}{$P$ value (F value) } \\
\hline & & & Interaction & Between groups & Within group \\
\hline \multicolumn{6}{|l|}{ FFD test - distance $(\mathrm{cm})$} \\
\hline PNF hamstring stretching & $6.03 \pm 11.09$ & $0.15 \pm 9.96$ & \multirow{2}{*}{$0.607(0.281)$} & \multirow{2}{*}{$0.929(0.008)$} & \multirow{2}{*}{$0.001^{*}(21.590)$} \\
\hline Jack-knife stretching & $6.29 \pm 13.09$ & $-.94 \pm 8.92$ & & & \\
\hline \multicolumn{6}{|c|}{ FFD test - anterior pelvic tilt $\left({ }^{\circ}\right)$} \\
\hline PNF hamstring stretching & $55.96 \pm 16.15$ & $66.99 \pm 12.76$ & \multirow{2}{*}{$0.240(1.542)$} & \multirow{2}{*}{$0.504(0.477)$} & \multirow{2}{*}{$<0.001^{*}(27.899)$} \\
\hline Jack-knife stretching & $62.76 \pm 19.39$ & $69.22 \pm 16.44$ & & & \\
\hline \multicolumn{6}{|l|}{ FFD test - lumbar flexion $\left(^{\circ}\right)$} \\
\hline PNF hamstring stretching & $96.93 \pm 13.07$ & $102.99 \pm 14.22$ & \multirow{2}{*}{$0.288(1.249)$} & \multirow{2}{*}{$0.883(0.023)$} & \multirow{2}{*}{$0.004^{*}(12.912)$} \\
\hline Jack-knife stretching & $96.09 \pm 19.25$ & $105.86 \pm 13.54$ & & & \\
\hline \multicolumn{6}{|l|}{ AKE test - right side $\left(^{\circ}\right)$} \\
\hline PNF hamstring stretching & $55.42 \pm 9.71$ & $64.83 \pm 7.15$ & \multirow{2}{*}{$0.340(0.994)$} & \multirow{2}{*}{$0.366(0.888)$} & \multirow{2}{*}{$<0.001^{*}(67.739)$} \\
\hline Jack-knife stretching & $52.67 \pm 10.55$ & $59.39 \pm 11.12$ & & & \\
\hline \multicolumn{6}{|l|}{ AKE test - left side $\left({ }^{\circ}\right)$} \\
\hline PNF hamstring stretching & $53.03 \pm 9.47$ & $61.78 \pm 8.94$ & \multirow{2}{*}{$0.957(0.003)$} & \multirow{2}{*}{$0.707(0.149)$} & \multirow{2}{*}{$<0.001^{*}(44.294)$} \\
\hline Jack-knife stretching & $54.61 \pm 8.46$ & $63.19 \pm 10.69$ & & & \\
\hline \multicolumn{6}{|l|}{ ASLR - right side $\left({ }^{\circ}\right)$} \\
\hline PNF hamstring stretching & $55.22 \pm 6.27$ & $68.42 \pm 6.85$ & \multirow{2}{*}{$0.990(<0.001)$} & \multirow{2}{*}{$0.558(0.364)$} & \multirow{2}{*}{$<0.001^{*}(118.973)$} \\
\hline Jack-knife stretching & $57.92 \pm 14.10$ & $71.08 \pm 10.99$ & & & \\
\hline \multicolumn{6}{|l|}{ ASLR - left side $\left(^{\circ}\right)$} \\
\hline PNF hamstring stretching & $56.50 \pm 9.45$ & $69.56 \pm 6.13$ & \multirow{2}{*}{$0.856(0.035)$} & \multirow{2}{*}{$0.699(0.158)$} & \multirow{2}{*}{$<0.001^{*}(152.390)$} \\
\hline Jack-knife stretching & $58.55 \pm 12.13$ & $71.06 \pm 11.44$ & & & \\
\hline
\end{tabular}

Data are expressed as mean \pm standard deviation.

Abbreviation: AKE, active knee extension; ASLR, active straight leg raising; FFD, finger-to-floor distance; PNF, proprioceptive neuromuscular facilitation.

${ }^{*} p<0.05$.

indicating that improved hamstring flexibility positively impacts pelvic movement during functional activities. Because hamstring muscles influence posterior pelvic tilt, ${ }^{2,3}$ theoretically, elongated hamstring muscles may allow more anterior pelvic tilt movement. However, we were surprised to find an increased lumbar flexion angle during the FFD test after the hamstring stretching exercises because we expected the increased anterior pelvic tilt to reduce lumbar flexion movements during forward bending activities. ${ }^{8}$ This finding may be related to our method for measuring lumbar flexion. In the present study, we calculated the lumbar flexion angle according to the position of the lumbar segment in the sagittal plane. Thus, the lumbar flexion angle could increase with the anterior pelvic tilt in the absence of lumbar movement. Therefore, an increase in anterior pelvic tilt movement may have influenced our findings regarding lumbar flexion during the FFD test.

Although some previous studies have reported that PNF hamstring stretching is superior to static hamstring stretching in terms of improving hamstring flexibility, ${ }^{5,18}$ our findings demonstrate that these hamstring stretching exercises have similar effects on hamstring flexibility. Although static stretching is generally performed in a passive manner, jackknife stretching was performed actively in the present study with contraction of the knee extensors. When the knee extensor muscles are activated, the knee flexors are relaxed because of reciprocal inhibition. ${ }^{14}$ Thus, compared to general static stretching, jack-knife stretching may have greater 
improvement effects on hamstring flexibility. Additionally, while isometric contraction of the hamstring muscles was held for $10 \mathrm{~s}$ in each trial in previous studies, ${ }^{16,18,20}$ our participants engaged in isometric contraction of the hamstring muscles for $8 \mathrm{~s}$ in each trial during PNF hamstring stretching. This procedural difference may have affected our results.

Our study has several limitations. First, we did not measure changes in muscle stiffness, alterations in the myotendinous junction, or sciatic nerve tension. ${ }^{23}$ Future studies may examine changes in these variables after PNF hamstring stretching and jack-knife stretching. Second, we calculated the lumbar flexion angle using global angle measurements but not relative angle measurements. Future studies may assess lumbo-pelvic movement using 3D motion analyses. Lastly, we did not consider that repetitions of measurements may influence results of AKE, ASLR, and FFD tests.

\section{CONCLUSIONS}

We compared the effects of PNF hamstring stretching and static stretching (i.e., jack-knife stretching) on hamstring flexibility and lumbo-pelvic movements. Our data show that both stretching exercises effectively increased knee extension and hip flexion ROM as well as anterior pelvic movement during a forward bending activity, despite no significant differences between the two groups. These findings indicate that PNF hamstring stretching and jackknife stretching have similar effects in terms of improved hamstring flexibility.

\section{Key Points}

Question Do hamstring stretching with proprioceptive neuromuscular facilitation (PNF) and jack-knife stretching have similar effects on hamstring flexibility?

Findings Both hamstring stretching exercises improved hamstring flexibility, and there were no differences in the effectiveness of the two exercises.

Meaning Both hamstring stretching with PNF and jack-knife stretching have similar beneficial effects on hamstring flexibility.

\section{Article information}

Conflict of Interest Disclosures: None.

Funding/Support: None.

Acknowledgment: None.

Ethic Approval: The study protocols were approved by the Institutional Research Review Committee of Inje
University.

\section{REFERENCES}

1. Kendall FP, McCreary EK, Provance PG, Rodgers MM, Romani WA. Muscles: testing and function with posture and pain. 5th ed. Baltimore, MD: Williams \& Wilkins; 2005.

2. Sahrmann SA. Diagnosis and treatment of movement impairment syndrome. St. Louis, MO: Mosby; 2002.

3. Neumann DA. Kinesiology of the musculoskeletal system: Foundations for rehabilitation. 3rd ed. St Louis, MO: Elsevier; 2017.

4. Mistry G, Vyas N, Sheth M. Comparison of hamstrings flexibility in subjects with chronic low back pain versus normal individuals. J Clin Exp Res. 2014;2(1):85.

5. Gunn LJ, Stewart JC, Morgan B, et al. Instrumentassisted soft tissue mobilization and proprioceptive neuromuscular facilitation techniques improve hamstring flexibility better than static stretching alone: a randomized clinical trial. J Man Manip Ther. 2019;27(1):15-23.

6. O'Hora J, Cartwright A, Wade CD, et al. Efficacy of static stretching and proprioceptive neuromuscular facilitation stretch on hamstrings length after a single session. J Strength Cond Res. 2011;25(6):1586-1591.

7. Meena V, Shanthi C, Madhavi K. Effectiveness of PNF stretching versus static stretching on pain and hamstring flexibility following moist heat in individuals with knee osteoarthritis. Int J Physiother. 2016;3(5):529-534.

8. Kang MH, Jung DH, An DH, Yoo WG, Oh JS. Acute effects of hamstring-stretching exercises on the kinematics of the lumbar spine and hip during stoop lifting. J Back Musculoskelet Rehabil. 2013;26(3):329-336.

9. Spernoga SG, Uhl TL, Arnold BL, Gansneder BM. Duration of maintained hamstring flexibility after a onetime, modified hold-relax stretching protocol. $J$ Athl Train. 2001;36(1):44-48.

10. Magalhães FEX, Junior ARDM, Meneses HTDS, et al. Comparison of the effects of hamstring stretching using proprioceptive neuromuscular facilitation with prior application of cryotherapy or ultrasound therapy. J Phys Ther Sci. 2015;27(5):1549-1553.

11. Kisner C, Colby LA. Therapeutic exercise: foundations and techniques. 6th ed. Philadelphia, PA: FA Davis Company; 2012.

12. Medeiros DM, Cini A, Sbruzzi G, Lima CS. Influence of static stretching on hamstring flexibility in healthy young adults: systematic review and meta-analysis. Physiother Theory Pract. 2016;32(6):438-445.

13. Shamsi M, Shahsavari S, Safari A, Mirzaei M. A ran- 
domized clinical trial for the effect of static stretching and strengthening exercise on pelvic tilt angle in LBP patients. J Bodyw Mov Ther. 2020;24(3):15-20.

14. Sairyo K, Kawamura T, Mase Y, et al. Jack-knife stretching promotes flexibility of tight hamstrings after 4 weeks: a pilot study. Eur J Orthop Surg Traumatol. 2013;23(6):657-663.

15. Oh JS, Kang MH. The effects of static hamstring stretching on hip motion and lumbo-pelvic kinematics. $J$ Int Acad Phys Ther Res. 2020;11(3):2102-2106.

16. Puentedura E, Huijbregts $P$, Celeste $S$, et al. Immediate effects of quantified hamstring stretching: hold-relax proprioceptive neuromuscular facilitation versus static stretching. Phys Ther Sport. 2011;12(3):122-126.

17. Gribble PA, Guskiewicz KM, Prentice WE, Shields EW. Effects of static and hold-relax stretching on hamstring range of motion using the FlexAbility LE 1000. J Sport Rehabil. 1999;8(3):195-208.

18. Yıldırım MS, Ozyurek S, Tosun O, Uzer S, Gelecek N. Comparison of effects of static, proprioceptive neuromuscular facilitation and Mulligan stretching on hip flexion range of motion: a randomized controlled trial. Biol Sport. 2016;33(1):89-94.

19. Singh AK, Nagaraj S, Palikhe RM. Neurodynamic sliding versus PNF stretching on hamstring flexibility in collegiate students: a comparative study. Int $J$ Phys Educ Sports Health. 2017;4(1):29-33.

20. Park S, Lim W. Effects of proprioceptive neuromuscular facilitation stretching at low-intensities with standing toe touch on developing and maintaining hamstring flexibility. J Bodyw Mov Ther. 2020;24(4):561-567.

21. Guissard N, Duchateau J, Hainaut K. Mechanisms of decreased motoneurone excitation during passive muscle stretching. Exp Brain Res. 2001;137(2):163-169.

22. Condon SM, Hutton RS. Soleus muscle electromyographic activity and ankle dorsiflexion range of motion during four stretching procedures. Phys Ther. 1987; 67(1):24-30.

23. Choi KH. Comparison of the anterior pelvic tilting angle during forward bending in individuals with and without sciatic nerve tension. J Musculoskelet Sci Technol. 2019;3(2):44-48. 